\title{
Through the Eyes to the Heart - Bilateral Non-Simultaneous Retinal Artery Occlusion in 33-Year-Old Female Probably Associated with a Paradoxical Embolism
}

\author{
Joanna Stachura $^{1 *}$, Joanna Stafiej ${ }^{1}$, Maria Bogdan ${ }^{2}$, Paweł Reisner ${ }^{1}$, Bartosz $Ł$ Sikorski ${ }^{1}$, Mateusz Burduk ${ }^{1}$, Joanna \\ Borucka $^{1}$ and Grażyna Malukiewicz ${ }^{1}$ \\ ${ }^{1}$ Department of Ophthalmology Collegium Medicum in Bydgoszcz, Nicolaus Copernicus University in Torun, Poland \\ ${ }^{2}$ Department of Cardiology Collegium Medicum in Bydgoszcz, Nicolaus Copernicus University in Torun, Poland
}

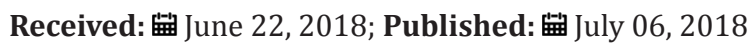

*Corresponding author: Joanna Stachura, Department of Ophthalmology Collegium Medicum in Bydgoszcz, Nicolaus Copernicus University in Torun, Poland

\begin{abstract}
Retinal artery occlusion (RAO) is uncommon before the age of 40 years. Bilateral RAO at young age are extremely rare. The etiology of RAO is usually associated with atherosclerotic risk factors and the presence of intravascular or intracardiac embolic material. In young adults, RAO may be manifestation of heart pathologies or inherited or acquired thrombophilia. We discuss characteristics, diagnostics and important clinical implications basing on the case of a previously healthy, non-smoking, non-hormonal therapy, a 33-year-old female with bilateral non-simultaneous RAO.

Keywords: Retinal Artery Occlusion; Paradoxical Embolism

Abbreviations: RAO: Retinal Artery Occlusion; BRAO: Branch Retinal Artery Occlusion; CRAO: Central Retinal Artery Occlusion; OCT: Optical Coherence Tomography; APTT: Activated Partial Thromboplastin Time; INR: International Normalized Ratio; ATIII: Antithrombin III; TTE: Transthoracic Echocardiography; TEE: Transesophageal Echocardiography; TCD: Transcranial Doppler; MTHFR: Methylenetetrahydrofolate Reductase; LA: Lupus Anticoagulant; aCL: Anti-Cardiolipin Antibodies; aB2GPI: Anti-Beta-2 Glycoprotein I Antibodies; ANA: Anti-Nuclear Antibody; ANCA: Antineutrophil Cytoplasmic Antibodies; ESR: Erythrocyte Sedimentation Rate; CRP: C-Reactive Protein; AFP: Alpha-Fetoprotein; CEA: Carcinoembryonic Antigen; B-HCG: beta-Human Chorionic Gonadotropin; CA 19-9: Cancer Antigen 19-9; ECG: Electrocardiography; TSH: Thyroid Stimulating Hormone; FT3: Free Triiodothyronine; FT4: Free Thyroxine; AST: Aspartate Aminotransferase; ALT: Alanine Aminotransferase; MRI: Magnetic Resonance Imaging; PFO: Patent Foramen Ovale
\end{abstract}

\section{Introduction}

RAO is an ophthalmic emergency causing acute ischemia of the area supplied by the vessel, thus resulting in retinal necrosis and partial or complete, with transient or persistent loss of vision acuity. It affects most commonly patients of over 60 years of age and it may concern 1/10 000 general population. RAO most often affects one eye but may be bilateral in $1-2 \%$ of cases. The etiology of retinal artery embolism is usually associated with carotid atherosclerosis (in the course of arterial hypertension, diabetes mellitus, hyperlipidemia or smoking addiction) and heart valve pathologies [1,2]. Thromboembolic diseases within the vessels of the eyes are rare in young people, with neither metabolic nor haemostatic abnormalities. In this paper particular attention was given to detailed diagnostics, through which it is possible to identify cardiovascular disorders, their proper treatment, which is associated with improved outcome in terms of another embolic incident and survival in this group of patients.

\section{Case Report}

A previously healthy, non-smoking, a 33-year-old Caucasian female presented to an emergency ophthalmological service with a sudden onset of painless, transient blurred vision in her right eye. She denied other systemic symptoms. There was no history of oral contraception use. The family history was negative. Ocular examination revealed best corrected visual acuity of 1,0 bilaterally. Biomicroscopy and intraocular pressure was normal in both eyes. A calcified white embolus was noted at the bifurcation of the superior temporal artery in her right eye together with mild retinal edema and pallor. No remarkable finding was observed in the posterior segment of the left eye. In OCT (DRI OCT Triton, Swept Source OCT; Topcon, Tokyo, Japan) without retinal changes in the macular area. Figure 1 shows the relevant retinal imaging studies. Visual field testing (Humphrey Field Analyzer; Carl Zeiss, Jena, Germany) disclosed an inferior temporal visual field defect 
in the right eye (Figure 2). With a diagnosis of right BRAO, the patient was treated with ocular massage, anti-glaucoma drops, pentoxifylline and anticoagulant and antiplatelet medications. On the first day of admission, the patient had an episode of vision loss in her left eye to hand motion. A relative afferent pupillary defect was identified on the left side. Examination of the left eye fundus revealed markedly slowed blood flow in narrowed retinal vessels, with "fragmentation" of the blood stream (cattle trucking) and whitening of the edematous retina surrounding pink central macula (cherry-red spot) (Figure 3A).

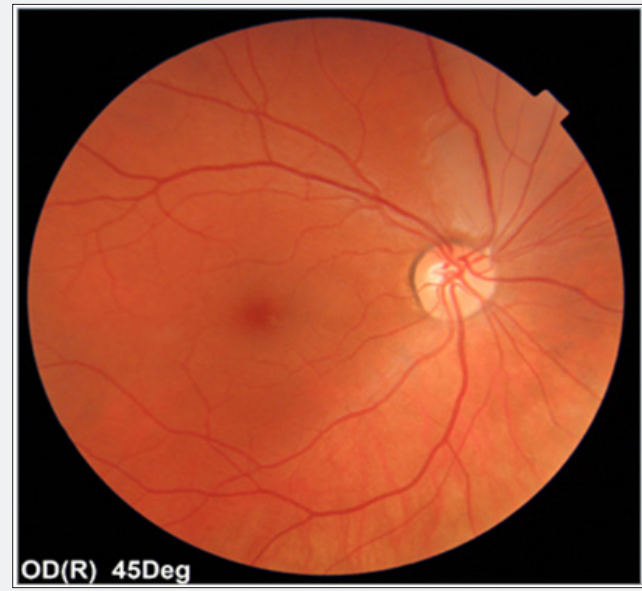

(A)

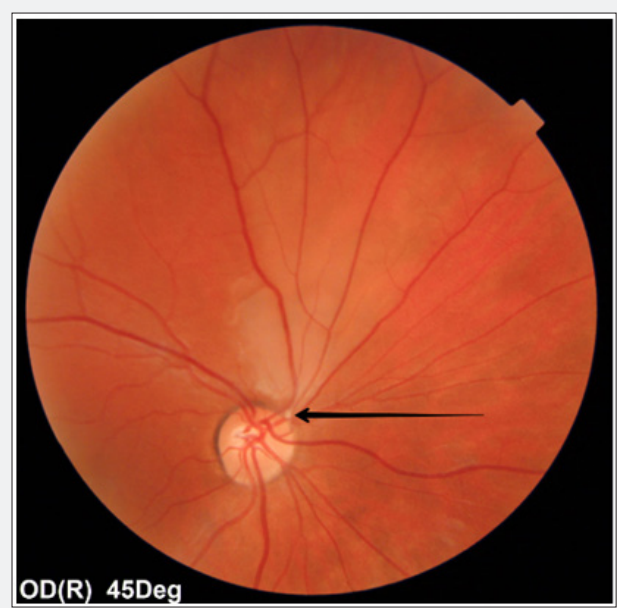

(B)

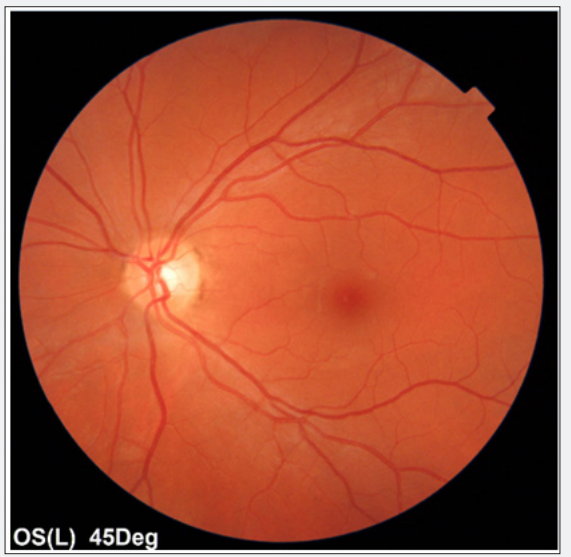

(C)

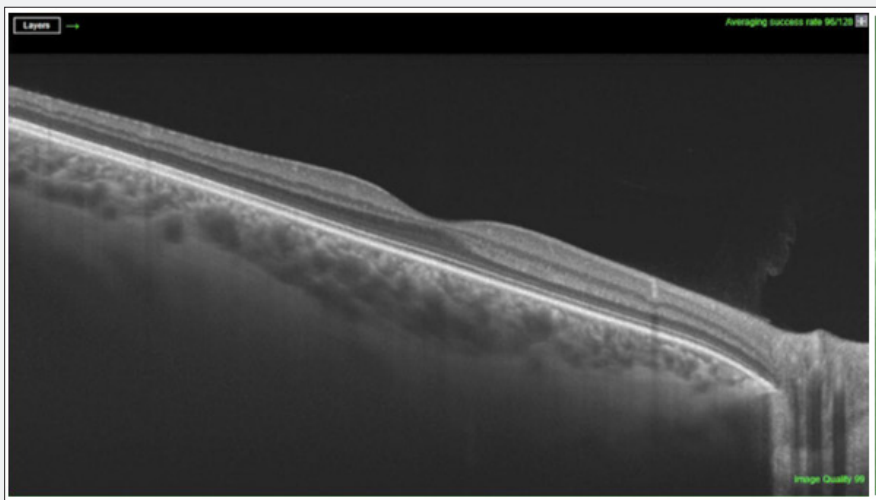

(D)

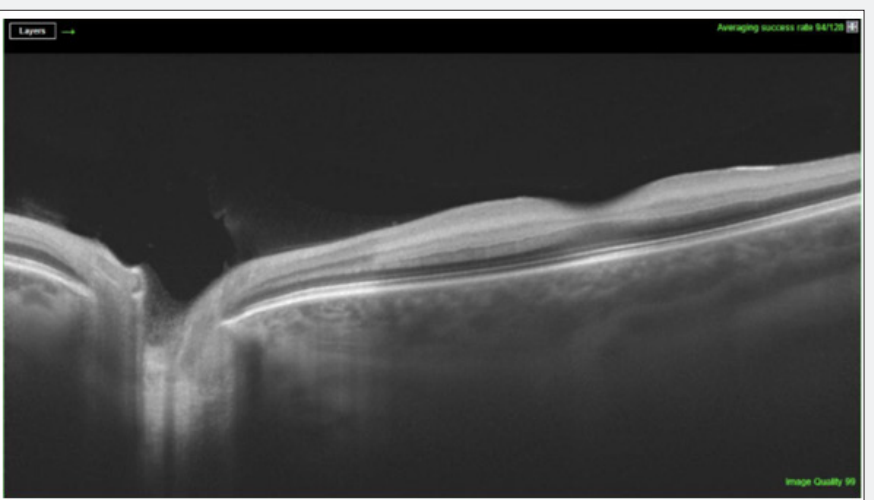

(E)

Figure 1: Colour fundus photographs of both eyes on admission.

(A) BRAO in right eye (mild whitening of the retina surrounding the superior temporal artery).

(B) Fundus photograph showing a retinal embolus (black arrow).

(C) Fundus image ofthe left eye in the age norm.

(D, E) Normal macular OCT in both eyes. 


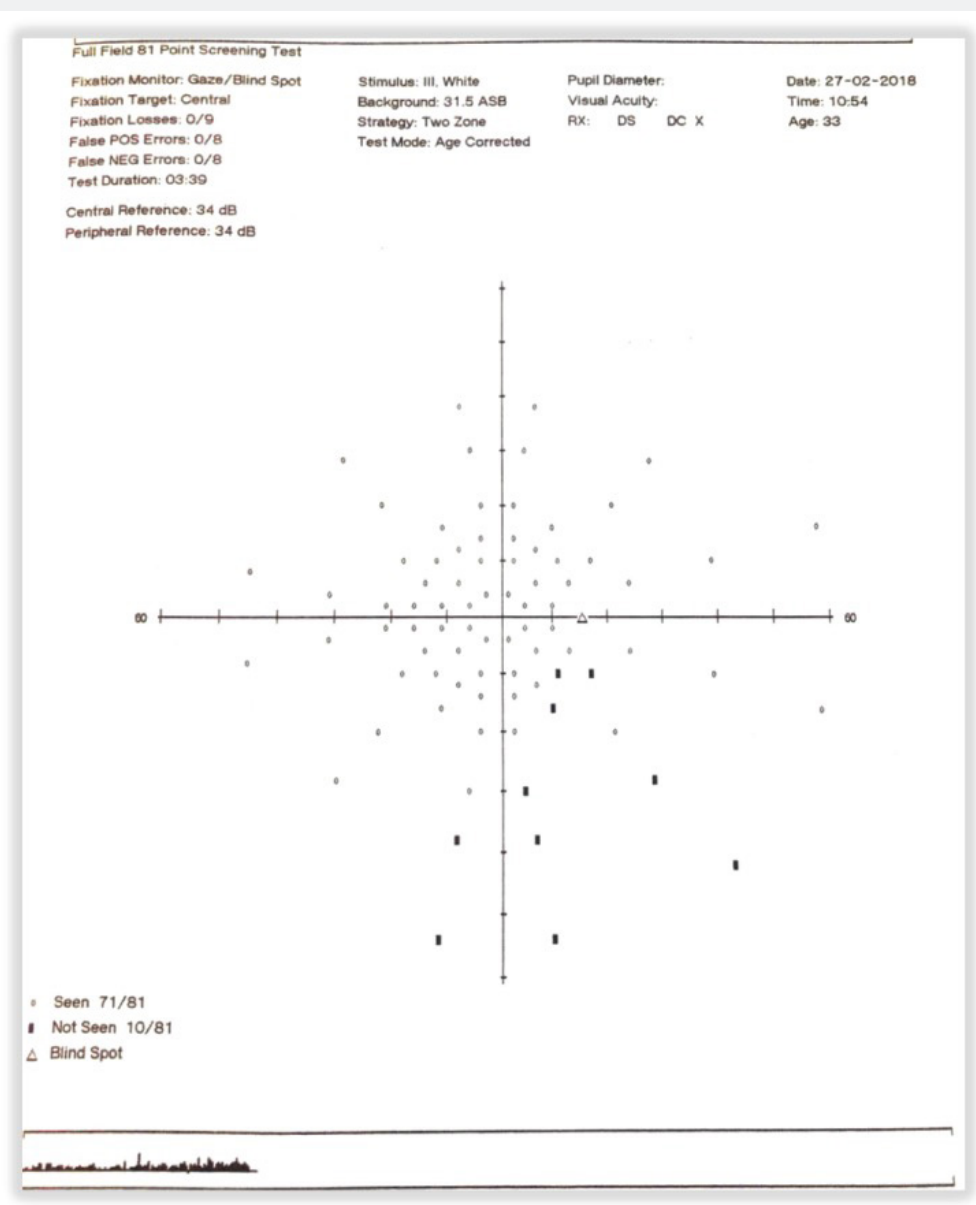

Figure 2: Full Field 81 perimetry of the right eye demonstrates a defect within the inferior temporal visual field.

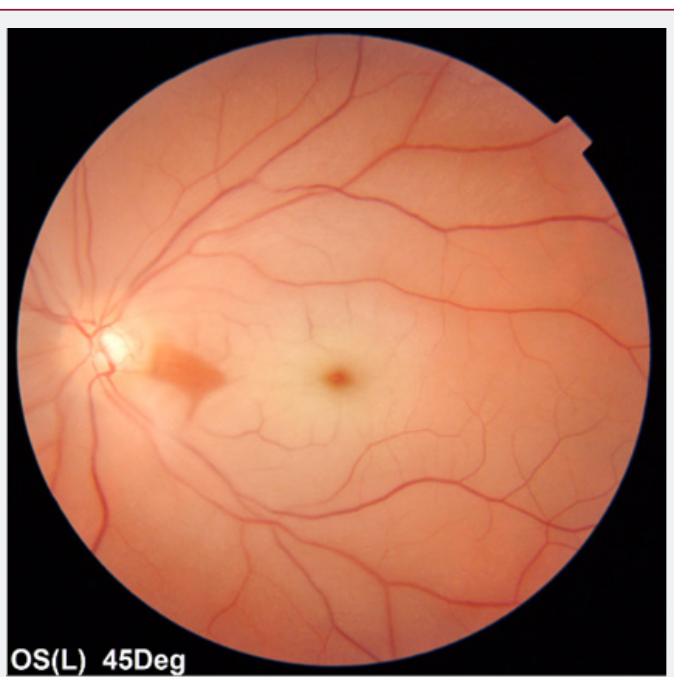

Figure 3: Imaging studies performed at the time of CRAO in the left eye.

(A) Fundus photography shows swelling of the macula with cherry-red spot.

OCT demonstrated an increased inner retinal layer thickness in the left eye (Figure 3B). CRAO of the left eye was diagnosed. An immediate left globe compression was performed with oral administration of acetazolamide. There was no significant improvement in left eye vision. The patient underwent an extensive diagnostic work-up consisting of neurology, cardiology, and hematology consultation. She was investigated for common vascular risk factor (blood pressure, glycemia, lipid profile, electrolytes, creatinine, APTT, INR, D-dimers, fibrinogen, ATIII), embolic sources (Duplex carotid ultrasound, Doppler veins of the 
lower limbs, TTE/TEE, TCD), hypercoagulable state (protein C\&S, homocysteine, factor VIII, factor IX, factor XI, molecular diagnostic testing for inherited thrombophilia: factor $\mathrm{V}$ Leiden, factor V R2, prothrombin, MTHFR polymorphisms C677T and A1298C, antiphospholipid antibodies: LA, aCL, aB2GPI), vasculitis (ANA, ANCA), myeloproliferative or sickle cell disease (morphology, blood film, ESR, CRP), neoplastic conditions (AFP, CEA, B-HCG, CA 19-9) and other (Holter ECG, TSH, FT3, FT4, ALT, AST, iron, ferritin, chest X-ray, abdominal ultrasound, MRI of the brain and orbits). TTE revealed mitral valve prolapse with a return wave. Based on the TEE, a PFO in the atrial septum was suspected. Due to the fact that the Valsalva maneuver could not be performed during TEE (PFO patency evaluation), it was decided to perform invasive diagnostics to check a right-to-left shunt occurrence. Cardiac catheterization before possible percutaneous PFO closure was planned. No other embolic sources were identified in the carotid artery, aortic arch, or heart.Genetic tests allowed detecting an abnormal CC homozygote for the 1298 mutation in the MTHFR gene. Other studies however, were unremarkable. Subsequently, the patient received long-term oral anticoagulant (under the control of INR) for the prevention of the next embolic event.

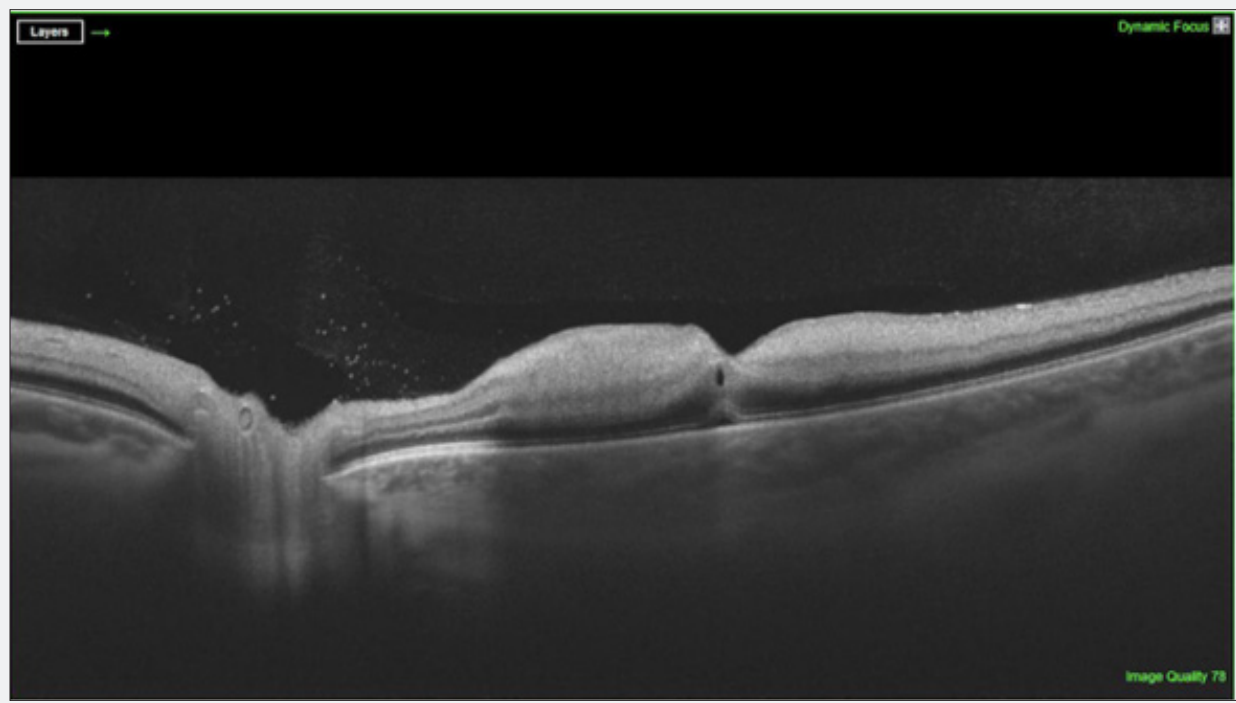

Figure 3B: Inner retinal thickening in OCT.

\section{Discussion}

Bilateral RAO at young age are extremely rare. Graven and colleagues [3] reported 21 patients (age range, 22 to 38 years) with RAO, of which 6 cases had bilateral BRVO. Hua et al. [4] described bilateral CRAO in 20-year-old female with systemic lupus erythematosus. Recently, Kinori et al. [5], however, have presented case of bilateral CRAO in a previously healthy 15-year-old girl. The mechanism for RAO before the age of 40 years is somewhat different than in patients of over 60 years of age because atheromatous vascular disease and its risk factors are typically absent [5]. In young adults, RAO may occur via multiple mechanisms, and investigations for systemic abnormalities are often exhaustive $[1,5]$. Our patient's history showed that bilateral non-simultaneous RAO was probably associated with a paradoxical embolism. Although routine diagnostic work-up including TTE were negative, TEE could establish a suspicion of a PFO. Kramer et al. [6] found TEE to have higher yield than TTE in the evaluation of patients with RAO [6]. As so far, few cases of PFO-related emboli have been published [7-9]. There was also weekly positive related factor, such as mutation in the MTHFR 1298 gene that can lead to elevated homocysteine in the blood (not in our patient), and thus be the cause of congenital hypercoagulability implicated as a risk factor for cardiovascular diseases. However, the role of this mutation in RAO pathogenesis remains controversial [10].

\section{Conclusion}

Proper diagnosis of RAO patients may allow to detect unrevealed cardiovascular disorders. At young age, RAO may be manifestation of heart pathologies, previously asymptomatic or the cause may be multifactorial. Due to the fact that half of potential retinal artery emboli visible in TEE are not detected in TTE, TEE also allows for the assessment of PFO, routine TEE in differential diagnosis of RAO is indicated in all patients without cardiovascular risk factors. This example underlines the importance of a multidisciplinary approach.

\section{References}

1. Varma DD, Cugati S, Lee AW, Chen CS (2013) A review of central retinal artery occlusion: Clinical presentation and management. Eye (Lond) 27(6): 688-697.

2. Hayreh SS, Podhajsky PA, Zimmerman MB (2009) Retinal artery occlusion: Associated systemic and ophthalmic abnormalities. Ophthalmology 116(110): 1928-1936.

3. Greven CM, Slusher MM, Weaver RG (1995) Retinal arterial occlusion in young adults. Am J Ophthalmol 120(6): 776-783.

4. Hua L, Patel K, Corbett JJ (2015) Bilateral central retinal artery occlusion in a patient with systematic lupus erythrematosus. J Stroke Cerebrovasc Dis 24(6): 139-141.

5. Kinori M, Simon SS, Kurup SP, Mets-Halgrimson R, Jampol LM, et al. (2017) Idiopathic bilateral central artery occlusion in a young woman. JAAPOS 21(5): 418-420. 
6. Kramer M, Goldenberg-Cohen N, Shapira Y, Axer-Siegel R, Shmuely H, et al. (2001) Role of transesophageal echocardiography in the evaluation of patients with retinal artery occlusion. Ophthalmology 108(8): 14611464.

7. Shoeibi N, Poorzand H, Abrishami M (2013) Branch retinal artery occlusion in patient with patent foramen ovale. J Ophthalmic Vis Res 8(1): 58-61.

8. Ballerini L, Cifarelli A, Ammirati A, Gimigliano F (2007) Patent foramen ovale and cryptogenic stroke: A clinical review. J Cardiovasc Med 8: 3438.

\section{ISSN: 2574-1241}

DOI: 10.26717/BJSTR.2018.06.001356

Joanna Stachura. Biomed J Sci \& Tech Res

(C) (i) This work is licensed under Creative

Submission Link: https://biomedres.us/submit-manuscript.php
9. Nakagawa T, Hirata A, Inoue N, Hashimoto Y, Tanihara H (2004) A case of bilateral central retinal artery obstruction with patent foramen ovale. Acta Ophthalmol Scand 82: 111-112.

10. Hickey SE, Curry CJ, Toriello HV (2013) ACMG Practice Guideline: Lack of evidence for MTFHR polymorphism testing. Genet Med 15(2): 153-156.

Assets of Publishing with us
BIOMEDICAL
RESEARCHES

